\title{
Ultrasound tomography for inline monitoring of plastic melts
}

\author{
Norbert Halmen, Christoph Kugler, Thomas Hochrein, Peter Heidemeyer, and Martin Bastian \\ SKZ - German Plastics Center, Würzburg, 97076, Germany \\ Correspondence to: Norbert Halmen (n.halmen@skz.de)
}

Received: 11 August 2016 - Revised: 30 November 2016 - Accepted: December 2016 - Published: 10 January 2017

\begin{abstract}
The inline determination of process and product parameters is of great help for the evaluation and optimization of new procedures. Therefore, an ultrasound process tomography system has been developed, which enables the imaging of the local filler distribution in plastic melts.

The objects investigated were extruded rods made of polypropylene (PP) with radial filler gradients. During extrusion, sound velocity and attenuation of the plastic melt were determined and processed via a modified reconstruction algorithm according to Radon transform into 2-D sectional images. Despite the challenges of higher attenuation and impedance mismatch of $60 \mathrm{~mm}$ filled PP melt compared to water, the resulting images are of good quality. An important factor for the image quality after tomographic reconstruction is the opening angle of the used ultrasound transducers. Furthermore, a simulation environment was developed in Matlab, which serves as a testing platform for the measurement system.
\end{abstract}

\section{Introduction}

\subsection{Ultrasound for process monitoring}

Ultrasound (US) waves are mechanical, acoustical - both shear as well as compressive - waves in the frequency range from $20 \mathrm{kHz}$ up to several $\mathrm{GHz}$, depending on the elastic and dissipative properties of the medium. There are two basic properties which are used for measurements. First, the speed of sound $c$ is given by Eq. (1),

$c=\frac{\Delta x}{\Delta t}$

where $\Delta x$ is the traveled distance and $\Delta t$ the propagation time. Second, the attenuation coefficient $\alpha$ can be defined by the intensity ratio of original $\left(I_{0}\right)$ and attenuated $(I)$ signal:

$\alpha=\frac{1}{2 \Delta x} \ln \frac{I_{0}}{I}$,

where the pulse intensity $I$ is

$I=\int_{t_{1}}^{t_{2}} A^{2}(t) \mathrm{d} t$.
Here, $A$ is the signal amplitude and $t_{1}$ and $t_{2}$ are the start and end time of an US pulse. In general, when examining materials with US, advantages can be taken of the frequencydependent attenuation due to viscous losses as well as scattering and thermoelastic effects while sound waves are propagating through a medium. Since US has been established mainly in the field of non-destructive testing and for diagnostic purposes in the medical sector, it has also arrived as a process and quality monitoring tool in the plastics sector. In liquids and plastic melts, US waves propagate mainly in the form of longitudinal waves. The material properties depend on the complex longitudinal modulus $M^{*}$, given by Eq. (4):

$M^{*}=M^{\prime}+i M^{\prime \prime}=\frac{\varrho c^{2}}{\left(1-\frac{i \alpha c}{\omega}\right)^{2}}$,

where $\varrho$ is the density, $c$ is the speed of sound, $\alpha$ is the attenuation coefficient and $\omega$ is the angular frequency, which is defined by

$\omega=2 \pi f$.

Since the attenuation in plastic melt is small in most cases, the real part of the longitudinal modulus can be simplified by

$M^{\prime}=\varrho c^{2}$. 
Then, the speed of sound $c$ can be easily calculated, which is composed of the real part of the bulk modulus $K^{\prime}$ and shear modulus $G^{\prime}$ :

$c=\sqrt{\frac{M^{\prime}}{\varrho}}=\sqrt{\frac{K^{\prime}+\frac{4}{3} G^{\prime}}{\varrho}}$.

Because of the very small shear modulus, Eq. (7) can be simplified in the following way:

$c=\sqrt{\frac{K^{\prime}}{\varrho}}$.

Equation (8) shows the pressure dependence of the speed of sound, since $K^{\prime}$ and $\varrho$ are pressure dependent. The generation of US waves is mostly based on the piezoelectric effect (Deutsch et al., 1997). Near-real-time measurements (Dinger, 2007) qualifies US as a non-destructive testing procedure for inline measurements in plastic melts.

In transmission arrangement (i.e., transmitter and receiver are arranged opposite to each other) the pulse is attenuated when passing through the melt and the propagation time depends on the polymer. This allows conclusions about the viscous and elastic properties of the plastic melt. Especially signals with frequencies between $100 \mathrm{kHz}$ and $10 \mathrm{MHz}$ can also propagate effectively in highly filled compounds (Alig et al., 2000) - but only as long as the size of the filler particles (scatterers) is much smaller than the wavelength. Previous studies have shown that the volume fraction of different filler materials in different base polymers can be determined by variations in sound velocity (Alig et al., 2000, 2005). However, this depends on pressure and temperature. In the same manner, the distribution of particles also has an influence on the attenuation of the US signal. Therefore, the filler size distribution and agglomerate formation can be determined as well (Alig et al., 2006; Schober et al., 2014; Wöckel et al., 2016).

Since piezoelectric materials are temperature sensitive they need to be protected from high temperatures. For this reason testing in hot media needs specially designed US test probes protected by appropriate measures (Putz et al., 2014). High temperature environments require delay lines which allow a thermal decoupling between the temperaturesensitive US transducers with their adhesive joints and the hot melt. Such delay lines are made of materials which possess good sound transmission but low heat conductivity properties, such as glass, ceramics, special steel alloys, or highperformance plastics (Deutsch et al., 1997). When sound waves propagate through interfaces between different materials the reflection coefficient $r$, defined as the ratio of reflected to incident amplitude of a sound wave, has to be considered. It is given by Eq. (9):

$r=\frac{Z_{1}-Z_{2}}{Z_{1}+Z_{2}}$, where $Z_{1}, Z_{2}$ are the material impedances of adjacent materials. The impedance of a material is calculated by

$Z=\varrho c$.

Equation (9) shows that impedance matching is very important to minimize reflection losses. This implies the importance of the right material choice. When working with polymer melts, materials like polyimide (PI) or polyetheretherketone (PEEK) can be used as coupling materials at temperatures of up to $250^{\circ} \mathrm{C}$.

\subsection{Polymeric gradient materials}

When extruding semi-finished plastic products, the subsequent component properties are intentionally modified through incorporating additives, for example, to fulfill special requirements on mechanical properties and resistance against light or chemicals. In this case, the functional additives are typically homogeneously distributed across the entire extruded profile. An intentional distribution of aggregates in the component can either be accomplished through co-extrusion in the form of sharply defined layer systems with concentration jumps on the boundary layers (Zhu et al., 2006) or through discontinuous procedures (Wu et al., 2004; Sun et al., 2008; Krumova et al., 2001). A continuous procedure for the extrusion of rods made of polypropylene (PP) with radial gradients of fillers is currently being developed (Hirt et al., 2015). As an optimal adjustment of the gradient (no local displacements, no agglomeration of the additives) is decisive for the component properties of the final semifinished product and allows unambiguous conclusions about the quality of the production process, the continuous monitoring of the extrusion process is of advantage. Typically, the examination is offline and takes place in the lab after production either visually by sample or microscope or with the use of X-ray computed tomography (CT). However, offline methods entail a long dead time in the area of hours between sample-taking (including cooling of the rod) and the presence of a measurement result, which makes the process development and optimization very time-consuming. Furthermore, very small differences in density (e.g., as in the case of polymer blends) cannot be resolved by X-ray CT.

\subsection{Ultrasound process tomography (USPT)}

For an inline determination and real-time process control an USPT system has been developed for an inline imaging of the melt during the extrusion of rods. In contrast to X-ray CT, US requires no safeguards and can be integrated into the process directly.

Comparable approaches already exist in the medical sector for mammographies (Schwarzenberg, 2009), in the plastic sector, for example, to dissolve the temperature distribution in a melt channel (Praher et al., 2014; Hopmann et al., 2016), or for non-destructive component testing (Haach and 
Ramirez, 2016). An US tomography-based application for spatially resolved determination of the filler distribution in plastic melts has not been reported so far.

\section{Experiment}

\subsection{Measurement system and materials}

The USPT system was designed to the geometry of rods and consists of a sensor ring with an inner diameter of $60 \mathrm{~mm}$, allowing an easy adoption on the melt channel right before the exit of the shaping nozzle (see Fig. 1). The used basic polymer was Sabic 505P, a PP with a density of $905 \mathrm{~kg} \mathrm{~m}^{-3}$ and a melt flow rate of $2 \mathrm{~g} / 10 \mathrm{~min}\left(230^{\circ} \mathrm{C}, 2.16 \mathrm{~kg}\right)$. For the fabrication of a black to white color gradient, two different fillers were used: on the one hand $0.18 \%$ carbon black (SR 301 Carbon Black of Sid Richardson Carbon \& Energy Co., particle size $30-50 \mathrm{~nm}$, density $1700-1900 \mathrm{~kg} \mathrm{~m}^{-3}$ at $20^{\circ} \mathrm{C}$ ) and on the other hand $2.2 \%$ titanium dioxide $\left(\mathrm{TiO}_{2} 2220\right.$ of Kronos Titan $\mathrm{GmbH}$, mainly rutile, particle size $150-250 \mathrm{~nm}$, density $4000 \mathrm{~kg} \mathrm{~m}^{-3}$ ). The longitudinal speed of sound in solid graphite is $1470 \mathrm{~m} \mathrm{~s}^{-1}$ at $20^{\circ} \mathrm{C}$ (Samsonov, 1968), and for titanium dioxide the value varies from $9070 \mathrm{~m} \mathrm{~s}^{-1}$ $\left(277^{\circ} \mathrm{C}\right)$ to $9240 \mathrm{~m} \mathrm{~s}^{-1}\left(27^{\circ} \mathrm{C}\right)$ (Munro, 2002). These values are significantly greater than the typical speed of sound for polymer melts of about $1000 \mathrm{~m} \mathrm{~s}^{-1}$. The melt temperature during processing ranged from 200 to $230^{\circ} \mathrm{C}$, and the pressure was around 1 bar. Moreover, measurements were conducted on distilled water.

The speed of sound in media depends on temperature. If the material parameters are known, the speed of sound can then be derived from (Hochrein and Alig, 2011)

$\vartheta_{\text {melt }}=C_{\vartheta}\left(c-v_{0}-C_{\mathrm{p}}(p-1 \mathrm{bar})\right)+\vartheta_{0}$,

with speed of sound $c$, melt temperature $\vartheta_{\text {melt }}$, medium specific reference temperature $\vartheta_{0}$, reference speed of sound $v_{0}$, temperature compensation coefficient $C_{\vartheta}$ and pressure compensation coefficient $C_{\mathrm{p}}$. As the pressure in our setup is close to 1 bar, Eq. (11) can be simplified to

$\vartheta_{\text {melt }}=C_{\vartheta}\left(c-v_{0}\right)+\vartheta_{0}$.

Using Eq. (12) and literature values for $C_{\vartheta}, \vartheta_{0}$ and $v_{0}$ for PP (Hochrein and Alig, 2011) for the temperature range from 180 to $260^{\circ} \mathrm{C}$, the speed of sound can be calculated as follows:

$c=\frac{\vartheta_{\text {melt }}-\vartheta_{0}}{C_{\vartheta}}+v_{0}=\frac{\vartheta_{\text {melt }}-473.15 \mathrm{~K}}{0.50 \frac{\mathrm{Ks}}{\mathrm{m}}}+954 \frac{\mathrm{m}}{\mathrm{s}}$.

Due to the material for the delay lines, the impedance mismatch at the interfaces to water and PP has to be taken into account. At $25^{\circ} \mathrm{C}$ the speed of sound is $2900 \mathrm{~m} \mathrm{~s}^{-1}$ and the temperature gradient is $-0.6\left(\mathrm{~m} \mathrm{~s}^{-1}\right) \mathrm{K}^{-1}$ and decreases linearly with increasing temperature. This correlation between

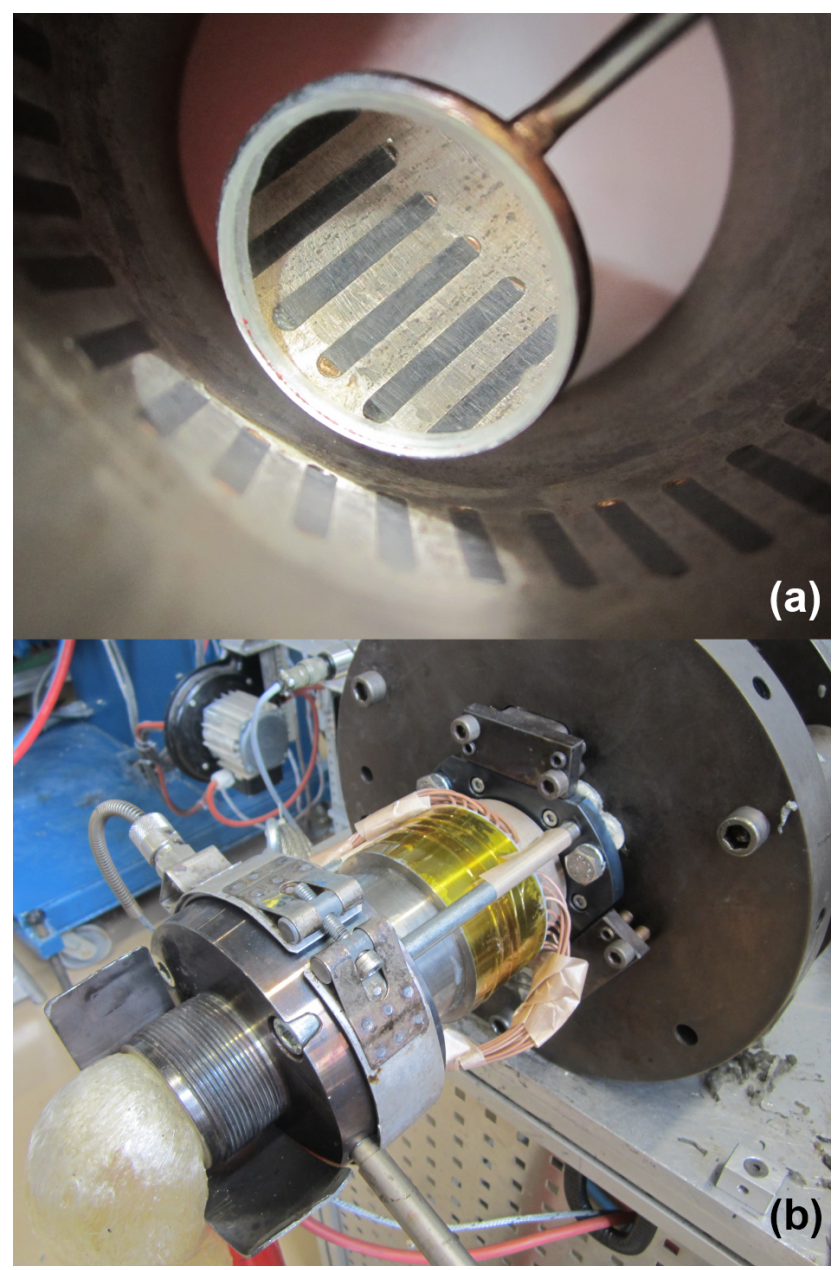

Figure 1. View on the delay lines of the US transducers inside of the USPT adapter (top) and USPT adapter (wrapped in yellow polyimide tape) mounted between gradient tool and shaping nozzle of the used extrusion line (bottom).

the speed of sound and the temperature for the delay line material is valid from 25 to $200^{\circ} \mathrm{C}$. The density of the material at $25^{\circ} \mathrm{C}$ is $1300 \mathrm{~kg} \mathrm{~m}^{-3}$. Since the high temperature material does not change its density in a wide range even at higher temperatures, the given density is used for the calculation of the impedance and reflection coefficients at $22^{\circ} \mathrm{C}$ for water and $220^{\circ} \mathrm{C}$ for PP, listed in Table 1 . It can be seen that for water the reflection losses are $17 \%$ lower due to the better impedance matching, resulting in higher signal intensities.

The attenuation coefficient of sound in water can be calculated using the k-Wave toolbox (Treeby and Cox, 2010) in Matlab. For a temperature of $22^{\circ} \mathrm{C}$ and a frequency of $2.12 \mathrm{MHz}$ the attenuation of water is calculated to $\alpha_{\mathrm{H}_{2} \mathrm{O}}=0.0093 \mathrm{~dB} \mathrm{~cm}^{-1}$. According to Schober et al. (2014) the attenuation coefficient for PP melt $\left(\alpha_{P P}\right)$ at the same frequency can be estimated to $0.3-0.6 \mathrm{~dB} \mathrm{~cm}^{-1}$ or even slightly higher because of higher temperature and lower pressure compared to the setup of Schober et al. (2014). Compared 
Table 1. Estimation of the reflection coefficients $r$ for the delay line-water interface and the delay line-PP melt interface at different temperatures $\left(\varrho\right.$ delayline $\left.=1300 \mathrm{~kg} \mathrm{~m}^{-3}\right)$.

\begin{tabular}{lrrrrrrr}
\hline Medium & $\begin{array}{r}T \\
\left({ }^{\circ} \mathrm{C}\right)\end{array}$ & $\begin{array}{r}\varrho \\
\left(\mathrm{kg} \mathrm{m}^{-3}\right)\end{array}$ & $\begin{array}{r}v \\
\left(\mathrm{~m} \mathrm{~s}^{-1}\right)\end{array}$ & $\begin{array}{r}Z \\
\left(10^{6} \mathrm{Ns} \mathrm{m}^{-3}\right)\end{array}$ & $\begin{array}{r}V_{\text {delayline }} \\
\left(\mathrm{m} \mathrm{s}^{-1}\right)\end{array}$ & $\begin{array}{r}Z_{\text {delayline }} \\
\left(10^{6} \mathrm{Ns} \mathrm{m}^{-3}\right)\end{array}$ & $r$ \\
\hline Water & 22 & 998 & 1489 & 1.49 & 2902 & 3.77 & 0.433 \\
PP & 220 & 905 & 994 & 0.900 & 2783 & 3.62 & 0.602 \\
\hline
\end{tabular}

to water, the attenuation coefficient of PP melt is about 30 to 60 times higher. The attenuation in the delay line material is specified with ca. $4 \mathrm{~dB} \mathrm{~cm}^{-1}$ for the given frequency.

Due to the higher impedance mismatch and higher signal attenuation of the plastic melt, high transmission powers of the US transducers are required to pass through a diameter of $60 \mathrm{~mm}$. For a good tomography imaging of the melt channel, each location point has to be scanned from as many directions as possible (Kak and Slaney, 2001). This can be accomplished either with a large number of transducers or with a wide radiation angle per transducer. With the given geometry, a large number of transducers would be synonymous with smaller apertures and therefore also larger opening angles. However, the maximum sound power per transducer decreases with its aperture due to the smaller contact surface to the melt.

The USPT was equipped with 40 equiangular transducers with plain apertures of $2 \mathrm{~mm} \times 14 \mathrm{~mm} \times 14 \mathrm{~mm}$ (width $\times$ length $\times$ depth), of which the delay lines are in direct contact with the melt (see rectangular insets in the adapter in Fig. 1). The narrow width orthogonally to the flow direction is to accomplish a greater opening angle in the sectional image plane of the tomography. The greater expansion along the flow direction of the melt causes only a very small aperture vertically to the sectional image plane but enlarges the contact surface of the transducers and thus the obtainable sound power per transducer. The delay lines were glued onto the piezo crystals using a two-component Epoxy adhesive. Figure 1 shows the sensor system from the inside as well as the adapter mounted to the gradient tool.

In contrast to typical tomographic methods, the projection angle is not varied by a mechanical rotation of transmitter and detector but electronically. The transducers in the different angular positions are successively set to transmitting mode while the other 39 transducers are in receiving mode. If the attenuation of the investigated medium (e.g., plastic melt) is high, up to four transducers can be bundled and used as a transmitter array. Through phase-delayed controlling of several transducers a focusing effect can be achieved and even a swivel of the sound field is possible.

The US transducers were driven with a rectangular windowed sinusoidal signal with a center frequency of $f=2.12 \mathrm{MHz}$ and a transmit voltage that can be changed stepwise from $12 V_{\mathrm{pp}}$ to $102 \mathrm{~V}_{\mathrm{pp}}$. Moreover, the number of burst cycles is also adjustable. Investigating water, the ex-

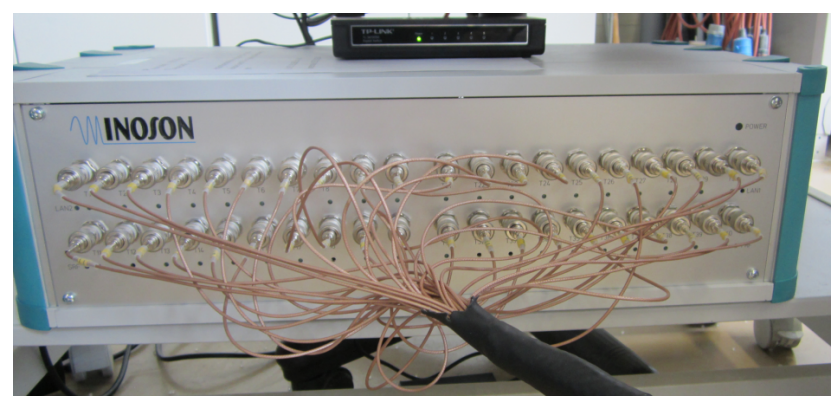

Figure 2. System electronics of the USPT system (Inoson PCM 12343).

periments were done with 1 or 3 bursts, while for PP melt 5 or even 10 bursts were used to achieve higher signal intensity. The US energy is emitted in the shape of a cone along the transducer axis. The maximum intensity is along the $0^{\circ}$ axis and decreases with increasing angle. Simulations done by the manufacturer resulted in about $6 \mathrm{~dB}$ lower signal intensity - so approximately only a quarter - at an angle of $30.3^{\circ}$. This value was defined as opening angle. The adapter and the transducers are designed for the production of gradient materials working at temperatures of up to $250^{\circ} \mathrm{C}$ and a pressure of up to 20 bar. Aside from the control of the transducers, the electronics of the system (Inoson PCM 12343, see Fig. 2) allows the collection of measurement signals with an ADC sampling rate of $12.5-100 \mathrm{MHz}$ and 256-16 384 sample points per acquired amplitude scan (A-scan). The gain level for every individual receiver is settable in 255 steps up to $80 \mathrm{~dB}$, and two $+12 \mathrm{~dB}$ software boosters can be used additionally. Fast functional testing and optimization of individual signal parameters were performed using the manufacturer software based on LabView. For tomographic measurements, beginning with signal acquisition through the analysis to the presentation of the results, a graphical user interface was developed using Matlab. The time to complete an entire measurement including all transducers with a sampling rate of $25 \mathrm{MHz}$ and 8192 sample points is approximately $8 \mathrm{~s}$. It has been found that these parameters were the best compromise of resolution and speed. 


\subsection{Calibration}

All delay lines for all US transducers in the system were produced manually, so geometric differences may occur. The length of the delay line in combination with its temperature dependence directly affects the measured sound velocity. In addition, the US transducers can exhibit different transmitting/receiving properties depending on the adherence of the piezo crystals to the delay line. Taking these factors into consideration, the system has to be calibrated accordingly. Distilled water was used as calibration medium since the speed of sound and the attenuation are well-known for defined temperatures.

For an absolute determination of the speed of sound within the USPT, the propagation time offset caused by the delay line of each transmitter and receiver pair (TRP) is necessary. With the measured distances between each TRP this time offset can be calculated for a given temperature. When using the system at higher temperatures it has to be adapted.

The calibration of the sound attenuation for this system is a bit tricky, since the transducers cannot directly be compared to one another by an opposing measurement setup. Therefore, a custom-made omnidirectional US transducer made by Inoson GmbH (see Fig. 3) was used in the center of the USPT system. The transducer has a diameter of $10.3 \mathrm{~mm}$ and was connected to a channel of the PCM. In transmission mode the transducer was driven with $2.12 \mathrm{MHz}$ and a transmit voltage of $47 V_{\mathrm{pp}}$, while in receiving mode a gain of $67 \%$ was set. Considering the intensities of the transmission pulse $I_{0}$ and echo (or reference) pulse $I$ along with their distances $x_{0}$ and $x\left(=3 x_{0}\right.$ in case of echo pulse) the US attenuation can be calculated using Eq. (2). Each individual US transducer of the USPT system is assigned with a corrective factor, which makes them comparable to one another. Using one transducer as a reference these corrective factors can be applied on the gain level settings. The material and impedance of the omnidirectional transducer are unknown, but since these corrective factors are only relative, those properties are not necessary. Once the gain levels have been set, also the angle dependence and pulse profile of every transducer can be determined.

\subsection{Reconstruction algorithm}

When using fillers, scattering effects at the particles has to be considered. Here the wavelength $\lambda$ is the decisive factor, which is defined as a quotient of speed of sound $c$ and frequency $f$ :

$\lambda=\frac{c}{f}$

For an US frequency of $f=2.12 \mathrm{MHz}$ and speed of sound of $c\left(220^{\circ} \mathrm{C}\right)=994 \mathrm{~m} \mathrm{~s}^{-1}$, the wavelength is about $\lambda=470 \mu \mathrm{m}$. Since the particle sizes given in Sect. 2.1 are smaller than $0.2 \lambda(94 \mu \mathrm{m})$ scattering is described by Rayleigh scattering. Therefore a ray-like propagation was assumed, where

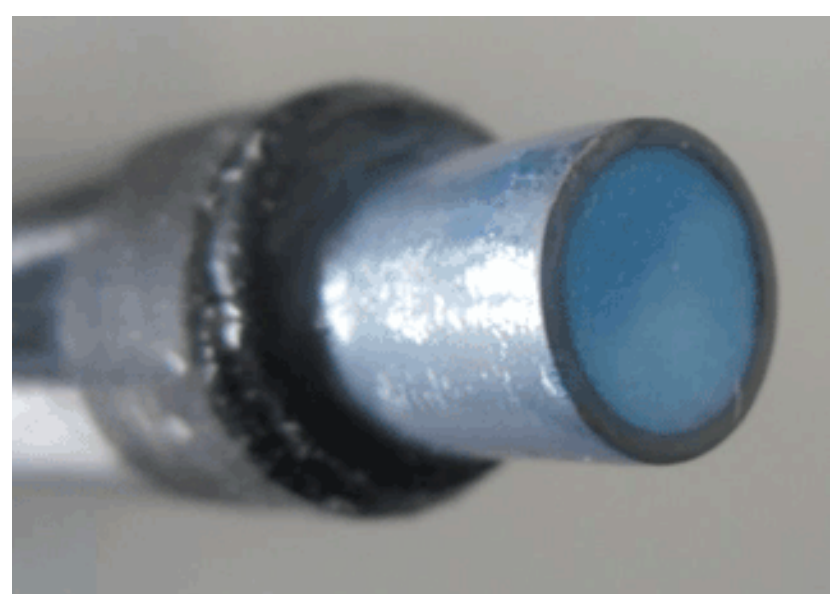

Figure 3. Custom-made omnidirectional US transducer made by Inoson $\mathrm{GmbH}$.

the wave on the direct way from the transmitter to receiver reaches the detector first. Scattered waves and reverberations are delayed and have smaller intensities. Furthermore, refraction and diffraction in polymer melts can be neglected in the first approximation. The used wave detection setup of a transmitter and opposing receivers is comparable to a fan beam with a certain opening angle. This was assumed for the calculation of the projections from different viewing angles.

To receive a sectional image from the individual projections, a classic back projection algorithm for a nondiffracting case according to Radon (Kak and Slaney, 2001) was adopted.

Only direct paths between transducers are considered, and the line integral for each TRP was calculated by

$$
L_{r, t}=\int_{\text {line }} f(x, y) \mathrm{d} s,
$$

where $f(x, y)$ is either the speed of sound or the attenuation at a certain pixel. In order to use the time-optimized function provided by Matlab for back projection reconstruction (inverse Radon transform) the fan beam geometry has to be converted into a parallel beam geometry (rebinning) since the function requires parallel beam projection data. Thereby, an image of all projections over one viewing angle is received. This is known as Radon transform or sinogram and defined by

$$
P_{\theta}(z)=\int_{-\infty}^{\infty} \int_{-\infty}^{\infty} f(x, y) \delta(x \cos \theta+y \sin \theta-z) \mathrm{d} x \mathrm{~d} y,
$$

where $\theta$ is the projection angle and $z$ the dimension of the projection. Because of the Fourier slice theorem, implying that the one-dimensional Fourier transform of a parallel projection is equal to a slice of the two-dimensional Fourier 
transform of the original object, we can estimate the object function by a two-dimensional inverse Fourier transform (Kak and Slaney, 2001).

With the inverse Fourier transform,

$f(x, y)=\int_{-\infty}^{\infty} \int_{-\infty}^{\infty} F(u, v) e^{i 2 \pi(u x+v y)} \mathrm{d} u \mathrm{~d} v$

where $F(u, v)$ is the Fourier transform of $f(x, y)$. Further details can be found in Kak and Slaney (2001). Equation (17) can be transformed to

$\left.f(x, y)=\int_{0}^{\pi} Q_{\theta}(w)(x \cos \theta+y \sin \theta)\right) \mathrm{d} \theta$,

with the projection $Q_{\theta}(w)$. Equation (18) shows that the projections over different angles are summed up to the object function, which is called back projection.

\subsection{Simulation}

Beside the measurement control and analyzation software, an additional simulation environment was developed in Matlab. Here, images, in which the different sound velocities and attenuation factors are depicted by different pixel values, are converted into matrices. The pixel values are integrated along the respective pixel line between a TRP. For the simulation the opening angle can be varied, which determines the number of opposing receivers used for reconstruction. By this, defective receivers can be simulated. In order to generate virtual data for the empty areas between the individual (and for defective) transducers the values were linearly interpolated. The reconstruction was done by creating 2-D sectional images with lines between TRPs of the integrated pixel value and subsequently summing them up. Afterwards a weighting function was applied, which took into account how often lines passed through a certain pixel.

The simulation enables the verification of real results, and the simulation algorithm can be improved on the basis of real measurement data. Thus, the suitability of the used USPT system can be determined beforehand for specific conditions (defective transducers, opening angle) and material systems. Moreover, the use of different corrective features, such as interpolation or filtering of the sinogram in the Fourier space (filtered back projections), can be examined.

\subsection{Measuring procedure}

The measuring procedure with real media is performed analogous to the simulation. Therefore, the same algorithm with some extensions concerning signal acquisition and data preprocessing is used. The measurement data are acquired with the PCM. The recorded A-scan signals (see Fig. 4) are bandpass filtered (finite impulse response) at the center frequency

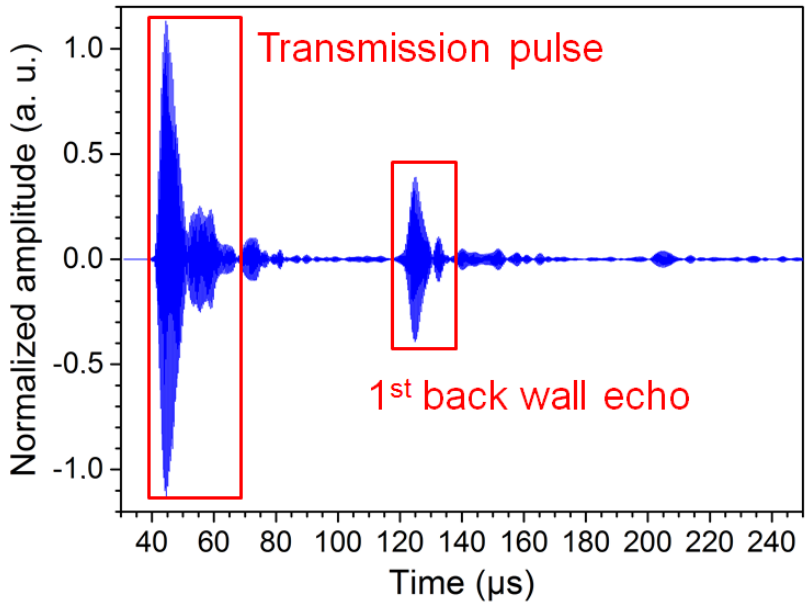

Figure 4. Bandpass-filtered A-scan of an US signal ( $53 V_{\mathrm{PP}}, 3$ burst cycles, gain level $49 \%$ ) of two opposing transducers in the USPT adapter filled with distilled water: the transmission pulse and the first back wall echo can be clearly distinguished and are marked in red.

$f$ with a relative bandwidth of $14 \%$ to suppress interfering noise signals. A main criterion was also to avoid overdriving the transducers and clipping by setting to high gain levels. Consequently, the propagation time is calculated for every signal impulse based on threshold detection algorithm, which indicates when the US pulse has reached the receiver. The threshold must be adjusted to the signal-to-noise ratio (SNR) depending on the medium. Since the melt in the adapter is very homogenous when the extrusion process has become stable, an improvement of the SNR through repeated measurements can be observed.

While only one transducer emits, every other transducer receives a signal. This allows the use of a high number of receivers for the reconstruction, resulting in a bigger opening angle. Initially, it has to be checked which receivers can be used for transmission. This can be done by comparing the maximum normalized signal amplitudes for every receiver while one transmitter is working. Figure 5 shows the comparison of the maximum normalized amplitudes of the receivers when transmitter 1 emitted for water and PP at different settings. Receiver 21 opposes transmitter 1. Based on several experiments, an opening angle of $60^{\circ}$ was chosen for the further work.

The propagation time together with the known distance of the TRP allows the calculation of the sound velocity along that particular path. The intensity of the pulse train is used to determine the attenuation. However, a measured reference pulse train is required to do so. This can either be a back wall echo (see Fig. 4) or a transmission pulse from a reference measurement. Optionally, by interpolation between measured values of adjacent receivers, virtual values can be generated for areas without US transducers or for defective transducers. Subsequently, the sinogram is created and a sec- 


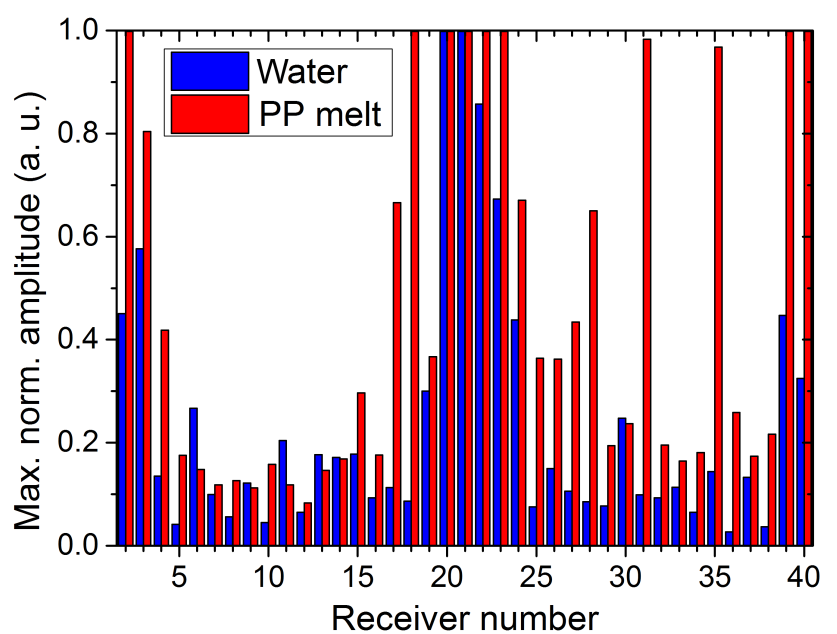

Figure 5. Comparison of maximum normalized signal amplitude for every receiver when transducer 1 emitted for water $\left(53 V_{\mathrm{PP}}\right.$, gain level all receivers 49\%, 3 burst cycles) and PP melt (102 $V_{\mathrm{PP}}$, gain level all receivers $69 \%, 10$ burst cycles, $+12 \mathrm{~dB}$ booster). The data presented here are raw data without corrective factors.

tional image can be reconstructed depicting the distribution of the sound velocity or the attenuation within the USPT system.

\section{Results}

\subsection{Simulation of agglomerates and gradients}

The effect of different opening angles of the transducers as well as the use of an interpolation on the reconstruction is exemplarily demonstrated on the basis of simulation images of agglomerates in Fig. 6 and a gradient of filler concentration offset from the symmetry axis in Fig. 7. The "original" pictures were created as grayscale images with maximum pixel values (255) for the agglomerates and the upper value of the gradients (the lowest was 0). The algorithm itself uses another color mapping. It can be perceived that the tradeoff made during the setup of the measurement system between number and aperture of the transducers leads to tomographic artifacts, such as ring artifacts, shadows, or blurring. These effects are removed through appropriate filtering and adjustments, improving the quality of the image.

The imaging resolution is limited by the width of the US transducers to $2 \mathrm{~mm}$. Due to the limited opening angles of the transducers the effective area for a meaningful reconstruction is restricted. The larger the opening angle the larger the area with good reconstruction results. Also the use of interpolation seemed helpful. Both effects are also demonstrated in Figs. 6 and 7.
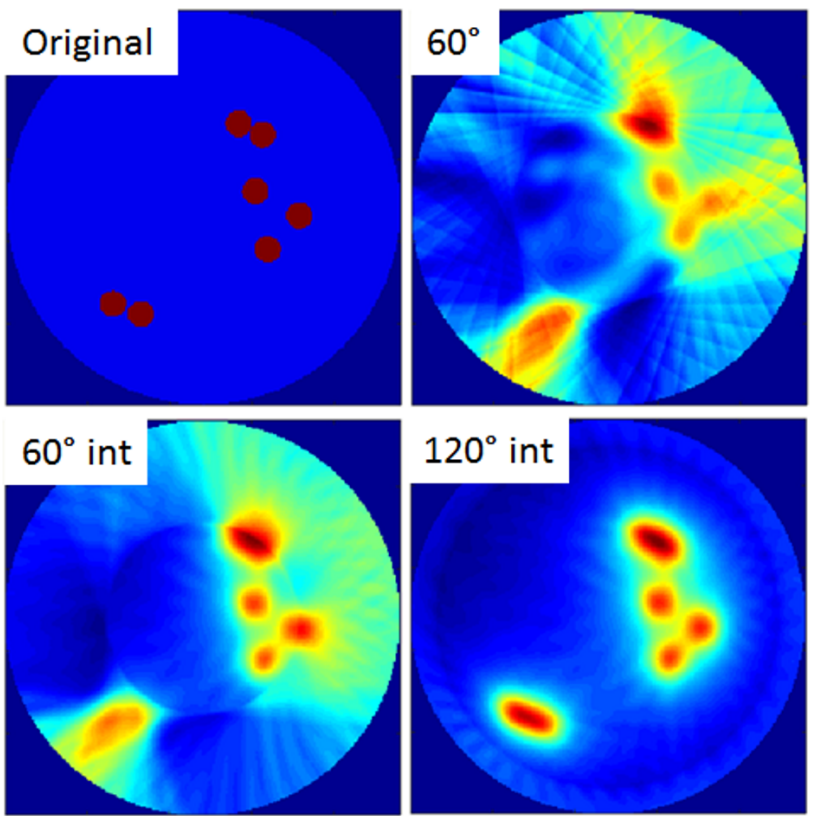

Figure 6. Simulation of a reconstruction of spatially distributed agglomerates for different opening angle and optional interpolation (int).
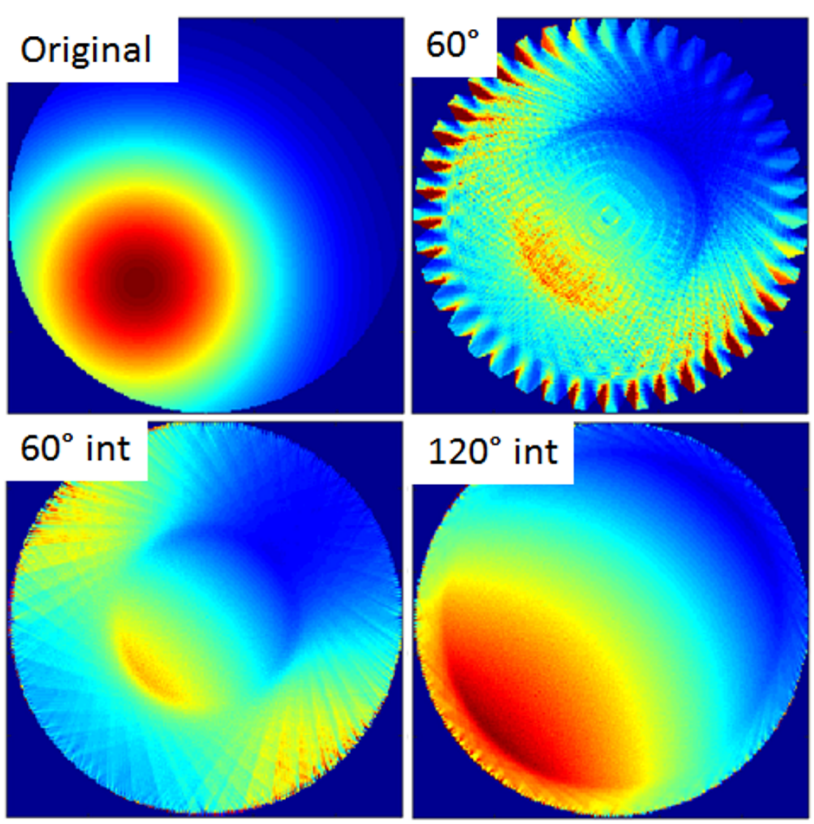

Figure 7. Simulation of a reconstruction of a de-centered gradient for different opening angle and optional interpolation (int).

\subsection{Measurements on filled PP melts}

The time for a single measurement with the typical settings mentioned in Sect. 2.1 is $0.2 \mathrm{~s}$ per transducer. For a full tomographic measurement using all 40 transducers, the whole measurement time sums up to $8 \mathrm{~s}$. Within the extrusion pro- 


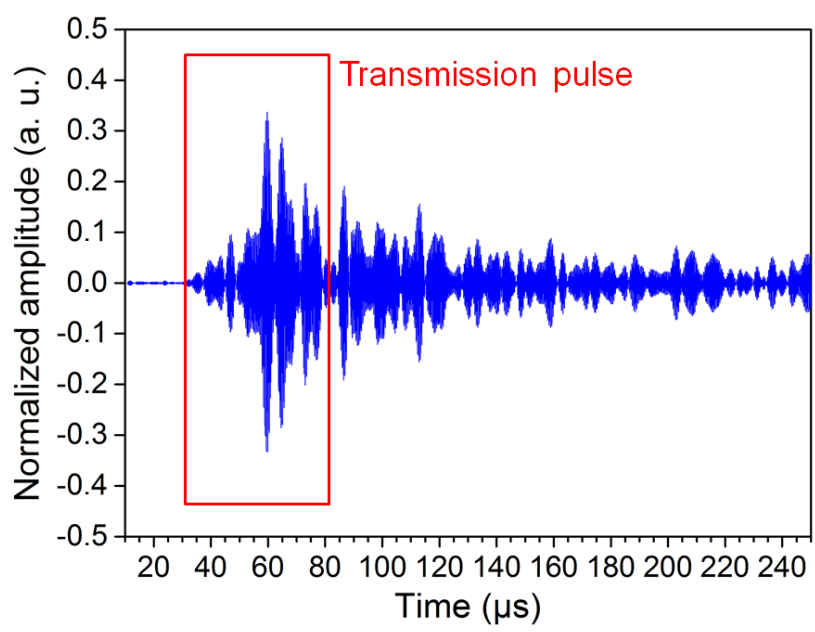

Figure 8. Bandpass-filtered A-scan of an US signal (102 $V_{\mathrm{PP}}$, 10 burst cycles, gain level $69 \%,+12 \mathrm{~dB}$ booster) of two opposing transducers in the USPT adapter of a PP melt filled with $\mathrm{TiO}_{2}$. The transmission pulse is marked in red.

cess, the polymer melt continuously flows during the measurement with a melt flow velocity of $v_{\text {melt }}=1.25 \mathrm{~mm} \mathrm{~s}^{-1}$. In this case the covered distance of the melt is about $1 \mathrm{~cm}$, thus $0.25 \mathrm{~mm}$ per active transmitter. Because of the movement during the measurement a Doppler effect has to be considered. The frequency shift $\Delta f$ is given by (Rosen and Gothard, 2010)

$\Delta f=\frac{v_{\text {melt }}}{c_{\mathrm{PP}}} f$,

with the center frequency $f$ and speed of sound in PP melt $c_{\text {PP. }}$. For the flowing PP melt can be calculated to $\Delta f=2.7 \mathrm{~Hz}$. Since the value is very small the Doppler effect and associated artifacts are considered negligible.

Nevertheless, homogeneity in the flow direction is essential to avoid motion artifacts in the reconstruction. As expected, measurements on PP melts showed more signal loss (impedance mismatch, attenuation) as well as more noise than in preliminary tests with water. As can be seen in Fig. 5, a higher maximum transmit voltage and a higher gain setting is necessary to achieve comparable signal amplitudes in PP to those in water. In this case, the pulse detection is distinctly more challenging. It is possible to penetrate $60 \mathrm{~mm}$ of filled PP melt, and a transmission pulse can still be detected clearly. But its length cannot be determined easily anymore because of noise and clutter. Accordingly, the first back wall echo is no longer detectable. Figure 8 shows an example of an A-scan of two opposing transducers measuring a PP melt filled with $\mathrm{TiO}_{2}$ and carbon black.

Moreover, the fact that only signals up to an opening angle of $60^{\circ}$ could be evaluated leads to lower quality in the outer region of the images. Figure 9 shows samples of different extruded rods with a radial filler gradient. As an example, Fig. 10 shows the experimental reconstruction of the sound

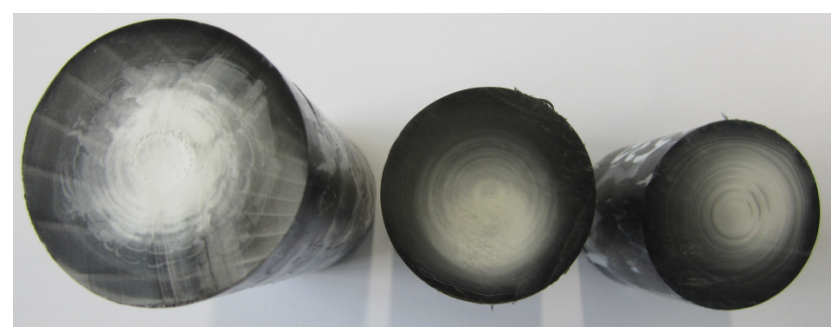

Figure 9. Graded filled rods $\left(\mathrm{PP}+\mathrm{TiO}_{2}+\right.$ carbon black), which had been examined using the USPT system in the melt.

velocity of pure PP melt as well as of filled melt with incorporated filler gradation. It can be clearly seen that the outer area is strongly affected by artifacts, especially after the incorporation of fillers. The influence of the fillers on the sound velocity also becomes apparent.

Unfortunately, the gradient cannot (yet) be determined as accurately as it actually can be created during the manufacturing process. Furthermore, an inhomogeneous temperature distribution cannot be excluded, even though the examination of pure PP melt exhibits a certain degree of homogeneity. The reconstruction of the attenuation also proves pretty difficult in the case of PP because the pulse trains cannot be defined clearly and scattering effects have a strong influence as well (see Fig. 8). Moreover, diffraction effects may occur when filler agglomerates are present in the direct path between a TRP.

\section{Discussion}

We have shown that the examination of the local filler distribution is possible with the USPT system. The geometry of the examined graded rods required a tradeoff between the number of transducers, transducer size, opening angle as well as achievable transmission power. As a result, the USPT system used here merely provides adequate image quality of the middle part in the reconstruction due to the limitation of the opening angle of maximum $60^{\circ}$. Efforts to perform real-time control require additional compromises between speed and reconstruction quality. The accuracy of propagation time detection using the current algorithm is $0.1-1 \mu$ s depending on the material system and the filler content. Limiting the signals to defined time frames yields time gains. This can be obtained by optimizing the sampling frequency and the number of sample points. However, this is at the expense of time resolution and the approximate signal propagation times need to be known. The spatial resolution is expected to be worse than $2 \mathrm{~mm}$, when assuming that a perfectly reconstructed point in the center of the USPT system can only be as small as the transducers. But this has to be verified in future work.

Filled PP melts could be penetrated with only one US transducer despite the $60 \mathrm{~mm}$ melt channel. However, due to impedance mismatch and increased signal attenuation, en- 

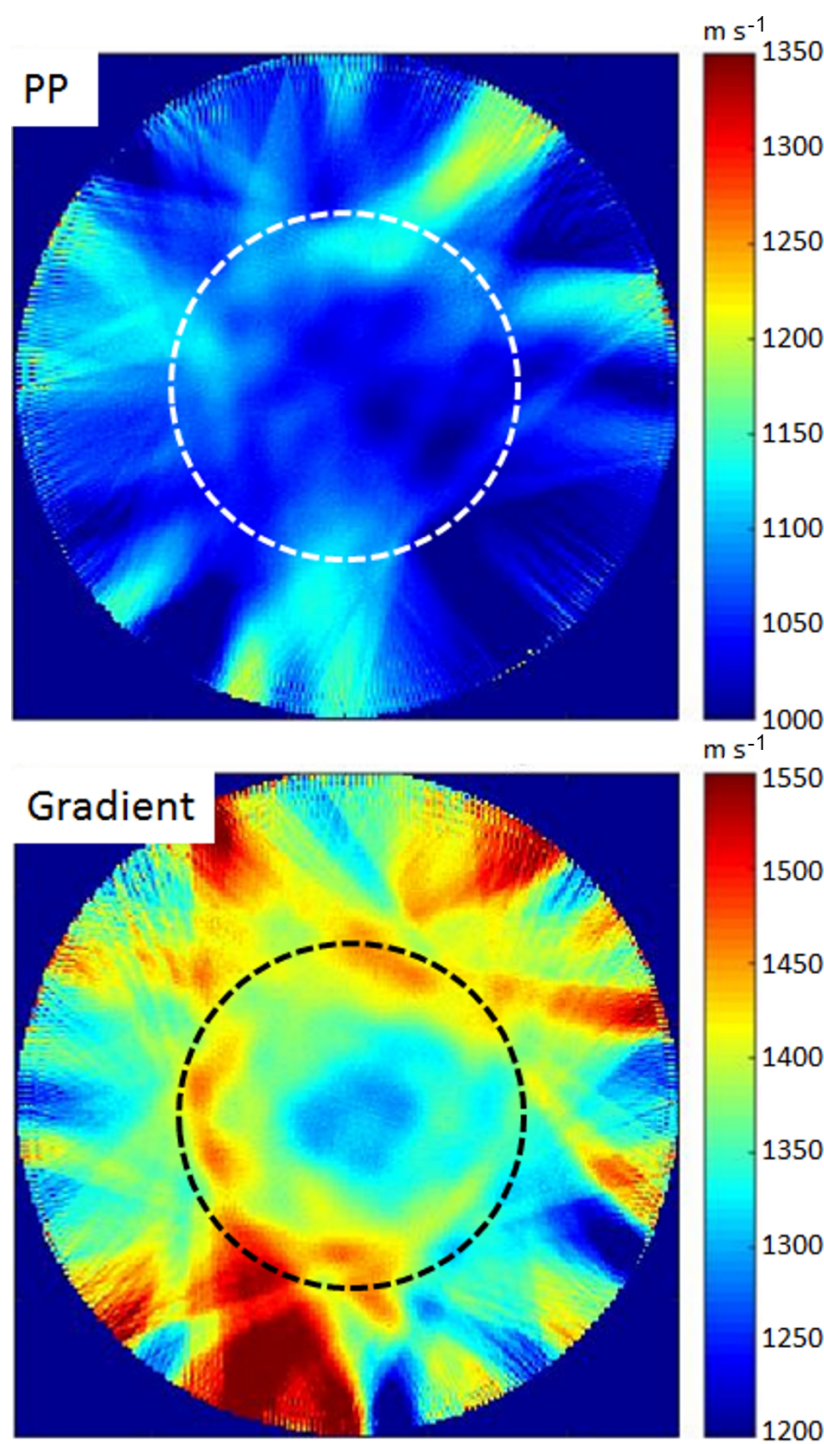
$\mathrm{m} \mathrm{s}^{-1}$

Figure 10. Reconstruction of the experimentally determined sound velocity of pure (top) as well as filled PP melts with radial filler gradient. Due to the opening angle of $60^{\circ}$, only the inner area of the image can be reconstructed satisfactory (dashed marking).

hanced signal amplification is required, which, however, also amplifies the noise. Therefore, a larger number of measurement repetitions are necessary to receive an adequate SNR.

The artifacts (rings, shadows) caused by the limited opening angles can only be removed partially through filtration and interpolation. Alternatively, an iterative reconstruction procedure could be used, which, however, requires more computing power. Advanced computing methods like algorithm parallelization and the use of multicore processors or graphic processing units can overcome this obstacle and, furthermore, even speed up the entire measurement.

The use of matched filter detection was discarded due to the 1600 TRP, which would have to be treated individually because of different manufacturing and different angles to each other. For the further development it surely offers the possibility to enhance the pulse detection. The creation of values between the receivers by linear interpolation proved to be a worthy tool, but it nevertheless needs to be verified in a specific setup with a dummy model. The evaluation of a reconstruction uncertainty is rather difficult at the moment. This is also a topic for upcoming experiments. Wave characteristics, diffraction and scattering will be taken into account for the next generation of the algorithm. Also an imaging for attenuation is planned. It also has to be investigated whether the filler concentration can be locally resolved. Finally, a reference measurement method needs to be developed which helps to validate the USPT system by being able to measure at the same system (or dummies) with a well-defined concentration distribution.

\section{Summary}

In this paper we have shown that it is principally possible to locally detect the spatially resolved distribution of fillers in plastic melts using an USPT system. Considering all influences either by factorization or corrections within the reconstruction algorithm, it is possible to depict the filler distribution in addition to the local temperature distribution. Composing the individual sectional images a full 3-D image of the rod can be generated subsequently. Thus, US tomography has great potential to open up further applications for inline process control of plastic melts. But there is still a well-filled agenda of things to do in further research work.

\section{Information about the Supplement}

The supplement contains the original image files used for the simulation as well as the raw data (Matlab files) of the measurements on water, PP melt and filled PP melt, which were used for this work.

\section{The Supplement related to this article is available online at doi:10.5194/jsss-6-9-2017-supplement.}

Acknowledgements. The research project VIII/3-3852/45/5 was funded by the Bavarian Ministry of Economic Affairs and Media, Energy and Technology. We would like to thank them for the financial support.

Edited by: J. Czarske

Reviewed by: four anonymous referees

\section{References}

Alig, I., Lellinger, D., Lamour, R., and Ramthun, J.: Inline Monitoring in the Extrusion Process Using Ultrasonics: Continuous 
Monitoring of Quality Parameters, Kunststoffe international, 5, 31-33, 2000.

Alig, I., Fischer, D., Lellinger, D., and Steinhoff, B.: Combination of NIR, Raman, Ultrasonic and Dielectric Spectroscopy for InLine Monitoring of the Extrusion Process, Macromol. Sy., 230, 51-58, doi:10.1002/masy.200551141, 2005.

Alig, I., Lellinger, D., Steinhoff, B., and Fischer, D.: Filler Dispersion: Ultrasonic Spectroscopy for In-Line Monitoring, Kunststoffe international, 5, 60-65, 2006.

Deutsch, V., Platte, M., and Vogt, M.: Ultraschallprüfung: Grundlagen und industrielle Anwendungen (ultrasonic testing: principals and industrial applications), Springer publishing house, Berlin, Germany, 1997.

Dinger, F.: Ultraschallverfahren für Prozesskontrolle und Qualitätsüberwachung (Ultrasonic method for process control and quality monitoring), Plastverarbeiter, 11, 92-94, 2007.

Haach, V. G. and Ramirez, F. C.: Qualitative assessment of concrete by ultrasound tomography, Constr. Build. Mater., 119, 6170, doi:10.1016/j.conbuildmat.2016.05.056, 2016.

Hirt, C., Lang, M., Kretschmer, K., Heidemeyer, P., and Bastian, M.: Grashalm als Vorbild - Gradientenwerkstoffe mit gezieltem Verlauf der Eigenschaftskennwerte (blade of grass as a model - gradient materials with targeted characteristics trend), Kunststoffe, 3, 109-112, 2015.

Hochrein, T. and Alig, I.: Prozessmesstechnik in der Kunststoffaufbereitung (process measurement techniques for plastics compounding), Vogel publishing house, Würzburg, Germany, 2011.

Hopmann, C., Spekowius, M., Wipperfürth, J., and Schöngart, M.: A concept for non-invasive temperature measurement during injection moulding processes, AIP Conf. Proc., 1, 1713, doi:10.1063/1.4942274, 2016.

Kak, A. and Slaney, M.: Principles of Computerized Tomographic Imaging, Society of Industrial and Applied Mathematics, doi:10.1137/1.9780898719277, 2001.

Krumova, M., Klingshirn, C., Haupert, F., and Friedrich, K.: Microhardness studies on functionally graded polymer composites, Compos. Sci. Technol., 61, 557-563, doi:10.1016/S02663538(00)00228-1, 2001.

Munro, R. G.: Elastic Moduli Data for Polycrystalline Ceramics, NISTIR, 6853, National Institute of Standards and Technology, Gaithersburg, Maryland, USA, 2002.
Praher, B., Straka, K., Usanovic, J., and Steinbichler, G.: Ultrasound based monitoring of the injection moulding process - Methods, applications and limitations, AIP Conf. Proc., 1593, 1, 159-162, doi:10.1063/1.4873755, 2014.

Putz, V., Burzic, I., Zagar, B., and Miethlinger, J.: UltraschallMessung von Strömungsprofilen in opaker Polymerschmelze während der Extrusion (Ultrasound measurement of velocity profiles in opaque polymer melt during extrusion), tm - Technisches Messen, 81, 371-380, doi:10.1515/teme-2014-1033, 2014.

Rosen, J. and Gothard, L. Q.: Encyclopedia of Physical Science, Facts on File, New York, 2010.

Samsonov, G. V.: Handbook of the physicochemical properties of the elements, IFI-Plenum, New York, USA, 1968.

Schober, G., Heidemeyer, P., Kretschmer, K., Bastian, M., and Hochrein, T.: Degree of Dispersion Monitoring by Ultrasonic Transmission Technique and Excitation of the Transducer's Harmonics, AIP Conf. Proc., 1593, 24-28, doi:10.1063/1.4873727, 2014.

Schwarzenberg, G.: Untersuchung der Abbildungseigenschaften eines 3D-Ultraschall-Computertomographen (investigation of the imaging properties of a 3D ultrasound computer tomograph), $\mathrm{PhD}$ thesis, Karlsruhe Series on Intelligent Sensor-Actuator-Systems, 6, KIT Scientific Publishing, doi:10.5445/KSP/1000011990, 2009.

Sun, C-K., Chen, L-J., and Chen, H-W.: Plastic wave-guide for terahertz wave, United States Patent, US 7409132 B2, 2008.

Treeby, B. E. and Cox, B. T.: k-Wave: MATLAB toolbox for the simulation and reconstruction of photoacoustic wave-fields, J. Biomed. Opt., 15, 021314, doi:10.1117/1.3360308, 2010.

Wöckel, S., Arndt, H., Steinmann, U., Auge, J., Dietl, K., Schober, G., Kugler, C., and Hochrein, T.: Statistical ultrasonic characterization of particulate filler in polymer compounds, 2016 IEEE International Ultrasonics Symposium (IUS), Tours, 1-4, doi:10.1109/ULTSYM.2016.7728747, 2016.

Wu, G., Wen, B., and Hou, S.: Preparation and structural study of polypropylene-talc gradient materials, Polym. Int., 53, 749-755, doi:10.1002/pi.1360, 2004.

Zhu, Y-B., Ning, N-Y., Sun, Y., Zhang, Q., and Fu, Q.: A new technique for preparing a filled type of polymeric gradient material, Macromol. Mater. Eng., 291, 1388-1396, doi:10.1002/mame.200600249, 2006. 